\title{
Clinical efficacy and survival analysis of apatinib
} combined with docetaxel in advanced esophageal cancer

This article was published in the following Dove Medical Press journal:

OncoTargets and Therapy

Jing $\mathrm{Li}^{*}$

Yongxu Jia*

Yaping Gao

Zhiwei Chang

Huiqiong Han

Jie Yan

\section{Yanru Qin}

Department of Oncology, The First Affiliated Hospital of Zhengzhou University, Zhengzhou 450052, Henan Province, People's Republic of China

*These authors contributed equally to this work
Correspondence: Yanru Qin Department of Oncology, The First Affiliated Hospital of Zhengzhou University, I Jian She East Road, Er qi District, Zhengzhou 450052, Henan Province, People's Republic of China Email yanruqin@163.com
Background and aim: Standard chemotherapy has limited clinical efficacy in patients with esophageal cancer and there is a significant and unmet clinical need for effective treatment options for these patients. The aim of this study was to compare the clinical efficacy of the novel, targeted drug apatinib combined with docetaxel, and docetaxel combined with S-1 as second- or further-line treatment for patients with advanced esophageal cancer.

Methods: We enrolled 33 patients with advanced esophageal cancer in chemotherapy group or apatinib combined with chemotherapy group in this retrospective study. Apatinib (500 mg) was taken orally once daily; docetaxel was administered at a dose of $75 \mathrm{mg} / \mathrm{m}^{2}$; and S-1 was optional at a dose of 40-60 mg, based on body surface area. The primary endpoint of this study was progression-free survival (PFS). Secondary endpoints included objective response rate (ORR), disease control rate (DCR), and the incidence and severity of adverse events (AEs).

Results: No complete response was observed in the two groups. However, two and five patients achieved partial response in the chemotherapy group and the apatinib combined with chemotherapy group, respectively. The ORR and DCR for the chemotherapy group was $11.1 \%$ and $33.3 \%$, respectively. In the apatinib combination group, ORR and DCR was $88.9 \%$ and $93.3 \%$, respectively. Anemia (11.1\%) and neutropenia (5.6\%) were the most frequent grade III/IV AEs observed in the chemotherapy group. In the apatinib combination group, the most frequent grade III/IV AEs were anemia (13.3\%), hypertension (6.7\%), and proteinuria (6.7\%). Median PFS was significantly longer in the apatinib combination group than in the chemotherapy group (175 days vs 85 days, $P=0.01$ ).

Conclusion: The combination of apatinib and docetaxel has a manageable toxicity profile and may prolong survival. Therefore, this combination may be used as as second- or further-line treatment for patients with advanced esophageal cancer.

Keywords: apatinib, esophageal carcinoma, vascular endothelial growth factor, survival analysis

\section{Introduction}

As one of the deadliest cancers worldwide, esophageal carcinoma, mainly divided into squamous cell carcinoma and adenocarcinoma, ranks sixth in mortality and in incidence, with 5-year survival rates of less than $20 \% .{ }^{1}$ Although diagnostic techniques have improved, some patients with esophageal cancer (ESC) are still initially diagnosed with late stage disease. Platinum-based chemotherapy and chemoradiation are standard-of-care treatments for patients with advanced disease not cured by surgery. However, overall patient survival remains limited despite numerous efforts to improve the clinical efficacy of chemotherapy. This deficiency highlights the urgent need for novel treatment strategies. Fortunately, the treatment of advanced cancer has entered 
a new era with the advent of targeted drugs in recent years. Targeted therapies have shown significant improvements in clinical efficacy and have also prolonged patient survival.

Angiogenesis has been demonstrated to be a multi-step hallmark of cancer. It is regulated by various factors and has been shown to be an important anti-cancer target. ${ }^{2,3}$ VEGF is the most critical driver of angiogenesis and there are three primary receptors, VEGFR-1, VEGFR-2, and VEGFR-3, which cooperate to initiate a signal transduction cascade in response to VEGF ligand binding. ${ }^{4}$ Previous studies have indicated that mice lacking VEGFR-2 were unable to form blood vessel systems and had fewer endothelial cells, ${ }^{5,6}$ revealing that VEGFR-2 may be essential in angiogenesis. VEGFR-2 has also been shown to regulate endothelial cell growth, differentiation, migration, and "tubulogenesis".

Bevacizumab, the VEGFR-2-targeting antibody, has exhibited significant antitumor activity in clinical trials and has been approved by the US Food and Drug Administration for the treatment of metastatic colorectal cancer (CRC) in combination with chemotherapy. ${ }^{8}$ Other VEGF-targeting agents, such as aflibercept, ${ }^{9}$ DW10075, are also being evaluated in ongoing clinical trials. ${ }^{10}$ In addition, small molecule tyrosine kinase inhibitors, such as sorafenib ${ }^{11}$ and sunitinib, ${ }^{12}$ have been applied to advanced cancers in succession. As a novel, selective inhibitor of VEGFR-2, ${ }^{13}$ apatinib (AiTan ${ }^{\mathrm{TM}}$, YN968D1, Hengrui Pharmaceutical Co. Jiangsu, People's Republic of China) has been verified as efficacious in a phase III clinical trial ${ }^{14}$ and was quickly approved to further-line treatment for advanced gastric cancer in 2014 by the China Food and Drug Administration. Subsequently, research has also indicated that apatinib alone or in combination with chemotherapy has potential antineoplastic activity in other malignancies, such as non-small-cell lung cancer, metastatic breast cancer, anaplastic thyroid cancer, CRC, and hepatocellular carcinoma. ${ }^{15-18}$ VEGFR has been shown to be expressed in approximately $53.8 \%$ of ESC patients and is associated with poor survival. ${ }^{19}$ However, clinical studies on the efficacy of apatinib treatment in ESC are really rare. In this study, compared to chemotherapy alone, we explored the relative clinical efficacy of apatinib combined with docetaxel in the treatment of ESC.

\section{Materials and methods Eligibility}

Patients with advanced ESC who were not eligible for surgery or radiotherapy, had failed first-line chemotherapy, had an Eastern Cooperative Oncology Group (ECOG) performance status (PS) of $\leq 1$, had no obvious abnormalities in heart, lung, liver, and kidney function, and can tolerate chemotherapy again were eligible for this study. The content and processes of the study have been reviewed and approved by the Ethics Committee of Zhengzhou University which is guided by international and national ethical requirements concerning biomedical research. This study was a retrospective study, which involved review of patients' medical histories. Therefore, patients' informed consent could not be obtained. In compliance with the Declaration of Helsinki, patient data were maintained with strict confidentiality.

\section{Study design}

Thirty-three patients were selected for this study between January 12, 2016 and February 16, 2018, at the First Affiliated Hospital of Zhengzhou University, 18 in the chemotherapy group, which received docetaxel (Qilu Pharmaceutical Co., Jiangsu, People's Republic of China) plus S-1 (Hengrui Pharmaceutical Co.), and 15 in the apatinib combination group, which received apatinib combined with docetaxel. Treatment continued until disease progression or intolerable toxicities were observed. Progression-free survival (PFS) was measured from the first day of administration to disease progression or death from any cause. Follow-up was carried out for all patients. The study endpoints were PFS, disease control rate (DCR), objective response rate (ORR), and the incidence and severity of adverse events (AEs). It is necessary to note that all 33 patients were previously treated with one of the following: cisplatin, carboplatin, oxaliplatin or other platinum and fluorouracil as first-line chemotherapy. Apart from the 33 patients who were included in the analysis, other patients were excluded from the study due to poor treatment compliance or economic hardship.

\section{Dose modification}

Patients received $500 \mathrm{mg}$ apatinib orally daily, and the dose of docetaxel and S-1 was calculated according to institutional standards based on body surface area (BSA). Docetaxel was administered intravenously at the standard Chinese dose of $75 \mathrm{mg} / \mathrm{m}^{2}$ every 3 weeks. Oral administration of dexamethasone at $8 \mathrm{mg}$ twice daily for 3 days was recommended as pre-medication for docetaxel infusion. S-1 was administered twice daily for 14 days followed by a 7-day drug holiday for each treatment cycle at the following doses based on BSA: $40 \mathrm{mg}$ (BSA $<1.25 \mathrm{~m}^{2}$ ), $50 \mathrm{mg}$ (BSA 1.25-1.5 $\mathrm{m}^{2}$ ), and $60 \mathrm{mg}\left(\mathrm{BSA} \geq 1.5 \mathrm{~m}^{2}\right.$ ). Doses were adjusted to $40 \mathrm{mg}$, $50 \mathrm{mg}, 60 \mathrm{mg}$, or $75 \mathrm{mg}$, according to the patient's condition. Treatment continued until disease progression or the incidence of intolerable toxicities. 


\section{Efficacy and safety assessment}

Tumors were evaluated regularly by computed tomography scans every two cycles according to Response Evaluation Criteria in Solid Tumors and classified as complete response, partial response, stable disease, or progressive disease. AEs were graded according to the Common Terminology Criteria for Adverse Events version 4.0.

\section{Statistical analysis}

SPSS 21.0 software was used for statistical analysis. Chi-squared test was used to compare the relationship between clinical characteristics of the two groups. The correlations between PFS and clinical characteristics were determined using the multivariate Cox-proportional hazards model. The Kaplan-Meier method was utilized to depict the survival curves. $P$-value $<0.05$ was considered statistically significant.

\section{Results}

\section{Patient characteristics}

As shown in Table 1, no significant differences were observed between the clinical features of the 33 patients in the two groups.

\section{Clinical efficacies}

Two partial responses were observed in the chemotherapy group, and five were observed in the apatinib combination group. Fourteen patients in the chemotherapy group and nine in the apatinib combination group also achieved stable disease. No complete response was observed in either group. Overall response rates of $11.1 \%$ and $33.3 \%$ were achieved in the chemotherapy and apatinib combination groups, respectively. Additionally, DCR was $88.9 \%$ in the chemotherapy group and $93.3 \%$ in the apatinib combination group. These results are summarized in Table 2.

Our results showed a significant difference in median PFS between the chemotherapy group ( 85 days) and the apatinib combination group (175 days) $(P=0.01)$. Based on multivariate Cox-proportional hazards regression analysis, the factor of treatment line may have prognostic significance $(P=0.008)$. In contrast, other factors such as gender ( $P=0.697)$, age ( $P=0.154)$, ECOG PS $(P=0.518)$, location of tumor $(P=0.477)$, pathologic type $(P=0.375)$, TNM stage $(P=0.519)$, and pleural effusion $(P=0.093)$ showed no significant correlation with PFS, as shown in Table 3.

Table I Clinical features of patients in the two treatment groups

\begin{tabular}{|c|c|c|c|c|}
\hline Characteristics & Total & $\begin{array}{l}\text { Chemotherapy } \\
\text { group }\end{array}$ & $\begin{array}{l}\text { Apatinib with } \\
\text { chemotherapy group }\end{array}$ & $P$-value \\
\hline Gender & & & & 0.722 \\
\hline Male & 13 & 8 & 5 & \\
\hline Female & 20 & 10 & 10 & \\
\hline Age (years) & & & & 0.482 \\
\hline$\leq 60$ & 14 & 9 & 5 & \\
\hline$>60$ & 19 & 9 & 10 & \\
\hline ECOG score & & & & 0.731 \\
\hline 0 & 21 & 12 & 9 & \\
\hline 1 & 12 & 6 & 6 & \\
\hline Location of tumor & & & & 0.917 \\
\hline Upper third & 9 & 5 & 4 & \\
\hline Middle third & 12 & 7 & 5 & \\
\hline Lower third & 12 & 6 & 6 & \\
\hline Pathologic types & & & & 0.512 \\
\hline Squamous cell carcinoma & 23 & 13 & 10 & \\
\hline Adenocarcinoma & 10 & 5 & 5 & \\
\hline TNM stage & & & & 0.703 \\
\hline Illa & 5 & 3 & 2 & \\
\hline Illb & 4 & I & 3 & \\
\hline Illc & 4 & 2 & 2 & \\
\hline IV & 20 & 12 & 8 & \\
\hline Treatment line & & & & 0.732 \\
\hline Second & 23 & 10 & 7 & \\
\hline Further & 10 & 8 & 8 & \\
\hline Pleural effusion & & & & $0.54 I$ \\
\hline Yes & 8 & 4 & 4 & \\
\hline No & 24 & 14 & II & \\
\hline
\end{tabular}

Abbreviation: ECOG, Eastern Cooperative Oncology Group. 
Table 2 Comparison of the short-term effects in the two treatment groups

\begin{tabular}{|c|c|c|c|c|c|c|c|}
\hline Group & $\mathbf{n}$ & CR & PR & SD & PD & ORR & DCR \\
\hline Chemotherapy & & & & & & & \\
\hline $\begin{array}{l}\text { Squamous cell } \\
\text { carcinoma }\end{array}$ & II & 0 & 1 & 9 & I & I (9.I) & $10(90.9)$ \\
\hline Adenocarcinoma & 7 & 0 & 1 & 5 & I & I (I4.3) & $6(85.7)$ \\
\hline $\begin{array}{l}\text { Apatinib and } \\
\text { chemotherapy }\end{array}$ & & & & & & & \\
\hline $\begin{array}{l}\text { Squamous cell } \\
\text { carcinoma }\end{array}$ & 10 & 0 & 5 & 4 & I & $5(50.0)$ & $9(90.0)$ \\
\hline Adenocarcinoma & 5 & 0 & 0 & 5 & 0 & $0(0.0)$ & $5(100.0)$ \\
\hline
\end{tabular}

Abbreviations: $C R$, complete response; PR, partial response; SD, stable disease; $\mathrm{PD}$, progressive disease; $\mathrm{ORR}$, objective response rate; $\mathrm{DCR}$, disease control rate.

\section{Evaluation of toxicity}

One patient in the chemotherapy group discontinued treatment after two cycles because of intolerable neutropenia, which resolved after treatment discontinuation. In the apatinib combination group, hypertension and proteinuria in two patients resulted in apatinib dose reduction from 500 to $250 \mathrm{mg}$.

The current study illustrated that the incidence of grade III/IV AEs was $22.2 \%$ and $46.9 \%$ in the chemotherapy and apatinib combination groups, respectively. In the chemotherapy group, grade III/IV AEs were seen in four patients and included neutropenia $(\mathrm{n}=1)$, anemia $(\mathrm{n}=2)$, and gastrointestinal hemorrhage $(\mathrm{n}=1)$. In the apatinib combination group, eight patients experienced the following AEs: neutropenia $(n=1)$, thrombocytopenia $(n=1)$, anemia $(n=2)$, hypertension $(n=1)$, proteinuria $(n=1)$, and gastrointestinal hemorrhage $(n=1)$. The incidence and severity of AEs are detailed in Table 4.

\section{Discussion}

ESC is one of the most common gastrointestinal tumors, with particularly high incidence in Linzhou, People's Republic of

Table 3 Cox multivariate analysis of clinical characteristics and PFS

\begin{tabular}{l|l|l}
\hline Characteristics & HR $(\mathbf{9 5} \% \mathbf{~ C l})$ & $\boldsymbol{P}$-value \\
\hline Gender & $1.238(0.423-3.62 I)$ & 0.697 \\
Age & $0.439(0.14 I-1.363)$ & 0.154 \\
ECOG score & $0.642(0.168-2.457)$ & 0.518 \\
Location of tumor & $1.279(0.649-2.522)$ & 0.477 \\
Pathologic type & $1.639(0.55 I-4.875)$ & 0.375 \\
TNM stage & $0.859(0.540-1.365)$ & 0.519 \\
Treatment line & $5.760(1.50 I-22.108)$ & $0.01 I^{*}$ \\
Pleural effusion & $0.263(0.055-1.249)$ & 0.093 \\
\hline
\end{tabular}

Note: $* P<0.05$.

Abbreviations: PFS, progression-free survival; ECOG, Eastern Cooperative Oncology Group.
Table 4 Toxicity profile based on treatment

\begin{tabular}{l|l|l|l|l}
\hline \multirow{2}{*}{ Toxicity } & \multicolumn{2}{l|}{$\begin{array}{l}\text { Chemotherapy } \\
\text { (n=I8), n (\%) }\end{array}$} & \multicolumn{2}{l}{$\begin{array}{l}\text { Apatinib and } \\
\text { chemotherapy } \\
\text { (n=I5), n (\%) }\end{array}$} \\
\cline { 2 - 5 } & I-II & III-IV & I-II & III-IV \\
\hline Neutropenia & I0(55.6) & I (5.6) & $7(46.7)$ & I $(6.7)$ \\
Thrombocytopenia & $9(50.0)$ & $0(0)$ & $5(33.3)$ & I (6.7) \\
Anemia & $8(44.4)$ & $2(I I . I)$ & $6(40.0)$ & $2(I 3.3)$ \\
Hypertension & I (5.6) & $0(0)$ & $3(20.0)$ & I (6.7) \\
Proteinuria & I (5.6) & $0(0)$ & $2(13.3)$ & I (6.7) \\
Hand-foot syndrome & $0(0.0)$ & $0(0)$ & I (6.7) & $0(0)$ \\
Vomiting/diarrhea & $8(44.4)$ & $0(0)$ & $4(26.7)$ & $0(0.0)$ \\
Fatigue & $4(22.2)$ & $0(0)$ & $3(20.0)$ & $0(0.0)$ \\
Gastrointestinal & $7(38.9)$ & I (5.6) & $3(20.0)$ & I (6.7) \\
hemorrhage & & & & \\
\hline
\end{tabular}

China. Numerous studies have illustrated that the occurrence, development, and progression of ESC involve an intricate multi-step process, which is regulated by several genes. Surgical treatment, radiotherapy, and chemotherapy are the main treatment methods for most cancers, including ESC. However, ESC is prone to recurrence, and hence, patients tend to have poor clinical outcomes. Consequently, there is currently no clinical consensus on treatment options for patients who have failed first-line chemotherapy or who have advanced or metastatic ESC.

Given the significance of angiogenesis in the development and progression of cancers, and the integral role of VEGFRs in pathological angiogenesis, VEGF-targeting has emerged as a viable therapeutic strategy for several malignancies. ${ }^{20-22}$ VEGFR-2 is functionally activated through autophosphorylation of its carboxyl terminus and subsequently stimulates PI3K-mediated cell survival and proliferation of endothelial cells. VEGFR-2 has also been shown to activate Src kinases to regulate a variety of biological responses, including cell proliferation, migration, differentiation, tumor microvascular density, and cell survival. ${ }^{7,23}$ Additionally, overexpression of VEGFR-2 has been demonstrated in several solid tumors. ${ }^{24}$

Apatinib, an oral, small-molecule tyrosine kinase inhibitor, is highly selective to bind to VEGFR-2 and exerts potent antitumor activity. The clinical efficacy of apatinib as monotherapy has been demonstrated in patients with: advanced melanoma with median PFS (mPFS) of 7.5 months, ${ }^{25}$ hepatocellular carcinoma with median time to progression of 4.8 months, ${ }^{26}$ metastatic CRC with mPFS of 3.82 months, ${ }^{18}$ and gastric cancer with mPFS of 2.8 months. ${ }^{14}$ Importantly, significant enhancement of overall survival (OS) and PFS was demonstrated in gastric cancer patients treated with a combination of apatinib and chemotherapy. ${ }^{27}$ Despite these 
recent successes, there are very few studies detailing the clinical efficacy of apatinib in patients with ESC. Therefore, we originally investigated the possibilities of antineoplastic activity caused by the combination of apatinib and chemotherapy for the treatment of patients with advanced ESC, who have failed first- or second-line chemotherapy.

Although no complete responses were observed in this study, ORR of $11.1 \%$ and $33.3 \%$ was observed in the

A

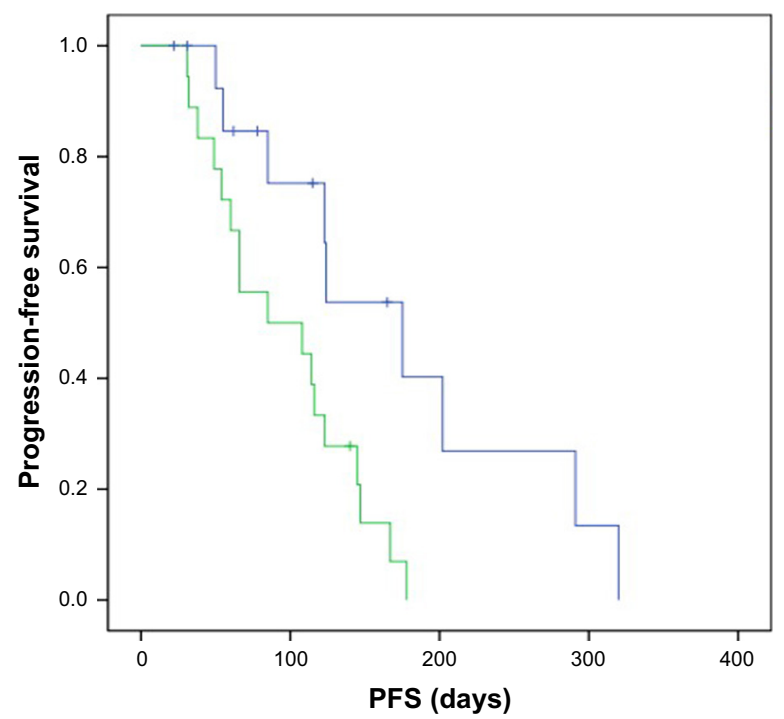

C

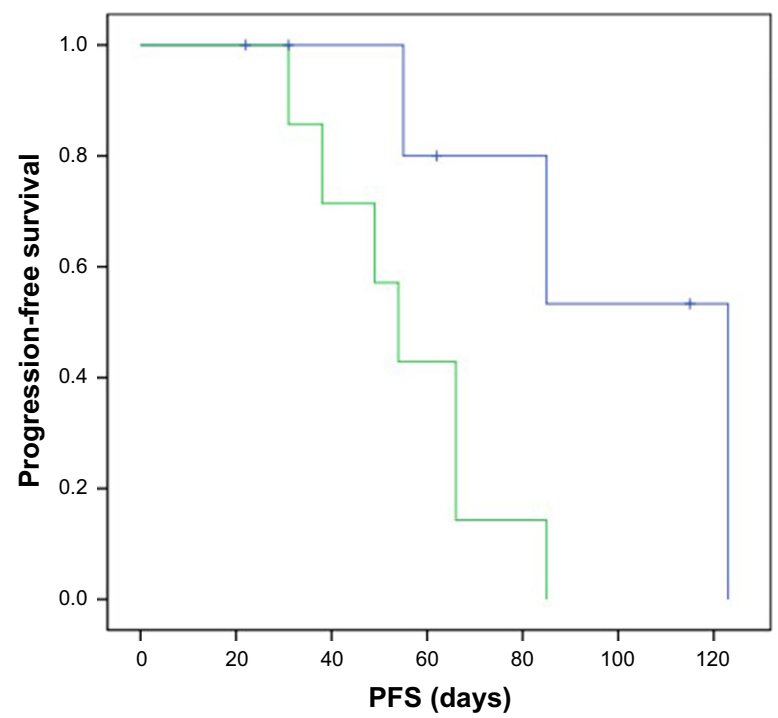

chemotherapy and apatinib combination groups, respectively. Additionally, DCR was $88.9 \%$ in the chemotherapy group and $93.3 \%$ in the apatinib combination group. Of note, mPFS was significantly higher in the apatinib combination group (175 days; 95\% CI 105.3-244.7 days) than in the chemotherapy group (85 days; 95\% CI 62.7-108.5 days) $\left(P=0.010\right.$, Figure 1A). A retrospective analysis ${ }^{28}$ of patients with advanced esophageal squamous cell carcinoma treated

B

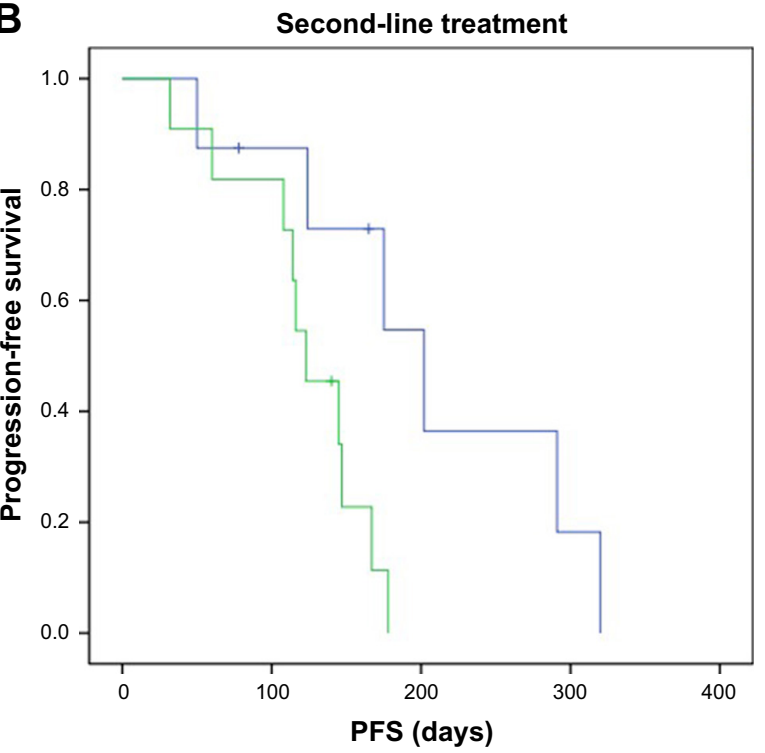

D

With pleural effusion

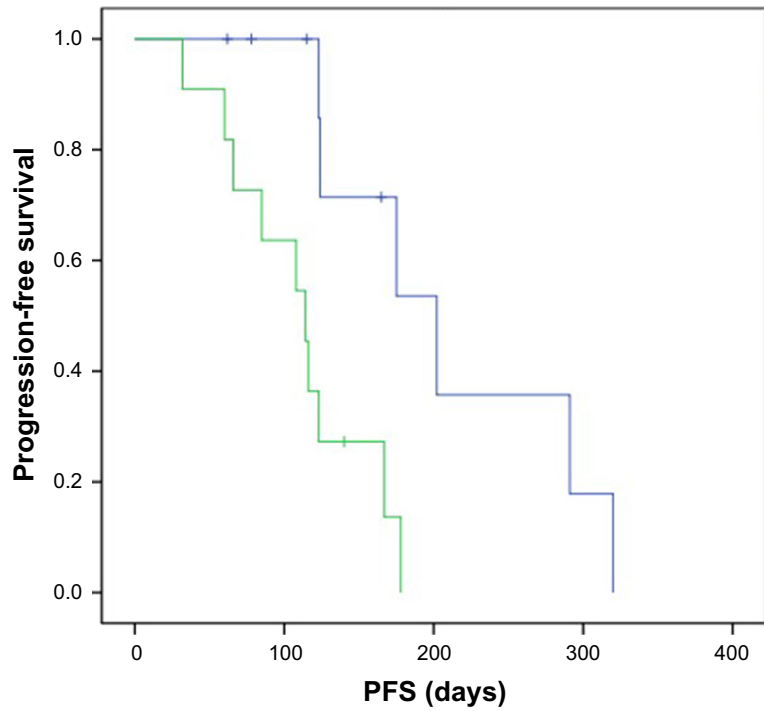

$$
\begin{aligned}
& \text { Groups } \\
& \neg \text { Apa+chemo }+ \text { Apa+chemo-censored } \\
& \neg \text { Chemo }+ \text { Chemo-censored }
\end{aligned}
$$

Figure I Progression-free survival (PFS) curves drawn by Kaplan-Meier method.

Notes: $(\mathbf{A})$ The overall comparison of PFS in the two treatment groups $(P=0.010)$. (B) The comparison of $P F S$ in the two groups regarding second-line treatment $(P=0.02 I)$. (C) The comparison of PFS in the two groups regarding third- or further-line treatment $(P=0.024)$. (D) The comparison of PFS in the two groups regarding patients with and without pleural effusion $(P=0.005)$.

Abbreviations: Apa, apatinib; chemo, chemotherapy. 
with apatinib alone showed mPFS of 115 days and OS of 209 days. The discrepancies might be due to the small sample size and the fact that patients enrolled were in better condition with lower ECOG scores, thus, our dates are slightly higher than those previously reported.

In addition, our study uncovered that the addition of apatinib to chemotherapy, regardless of second- or third-line regimen, could significantly prolong mPFS (202 days vs 123 days, $P=0.021 ; 123$ days vs 54 days, $P=0.024$; Figure $1 \mathrm{~B}$ and $\mathrm{C}$ ). There was also a significant improvement in mPFS in patients without pleural effusion in comparison to patients with pleural effusion (202 days vs 114 days, $P=0.005$; Figure $1 \mathrm{D} ; 55$ days vs 54 days, $P=0.892$ ), indicating that earlier treatment with apatinib may be more beneficial for patients with advanced ESC, particularly for those without pleural effusion.

In the current study, there were differences observed in the incidence of grade III/IV AEs observed in both groups. In particular, anemia (11.1\%), neutropenia (5.6\%), and gastrointestinal hemorrhage $(5.6 \%)$ were most frequent in the chemotherapy group. In contrast, hypertension $(6.7 \%)$, proteinuria (6.7\%), and gastrointestinal hemorrhage $(6.7 \%)$ were frequently observed in the apatinib combination group. These data are consistent with previous reports. ${ }^{29}$ It has been shown that hypertension and hand-foot skin reaction were correlated with longer mPFS and OS, and may serve as predictors of the clinical efficacy of apatinib in patients with breast cancer. ${ }^{30}$ This result has not been validated in other clinical trials, and our study was not powered to conduct in-depth analysis.

In summary, apatinib was investigated for valuable clinical advantages with a tolerable safety profile in patients with advanced ESC in the present study. The results suggest that apatinib might become an alternative and viable approach for second- or further-line regimens and it might be particularly beneficial, to a large extent, for the survival of patients with advanced ESC. Large-scale, prospective, randomized clinical studies are needed to validate and expand the findings of our study.

\section{Acknowledgments}

This work was supported by a grant from the National Natural Science Foundation of China (number 81472605). We thank LetPub (www.letpub.com) for its linguistic assistance during the preparation of this manuscript.

\section{Disclosure}

The authors report no conflicts of interest in this work.

\section{References}

1. Jemal A, Bray F, Center MM, Ferlay J, Ward E, Forman D. Global cancer statistics. CA Cancer J Clin. 2011;61(2):69-90.

2. Ferrara N, Kerbel RS. Angiogenesis as a therapeutic target. Nature. 2005;438(7070):967-974.

3. Risau W. Mechanisms of angiogenesis. Nature. 1997;386:671.

4. Yancopoulos GD, Davis S, Gale NW, Rudge JS, Wiegand SJ, Holash J. Vascular-specific growth factors and blood vessel formation. Nature. 2000;407:242.

5. Shalaby F, Rossant J, Yamaguchi TP, et al. Failure of blood-island formation and vasculogenesis in Flk-1-deficient mice. Nature. 1995; 376:62.

6. Carmeliet P, Ferreira V, Breier G, et al. Abnormal blood vessel development and lethality in embryos lacking a single VEGF allele. Nature. 1996;380:435.

7. Rahimi N. Vascular endothelial growth factor receptors: Molecular mechanisms of activation and therapeutic potentials. Exp Eye Res. 2006;83(5): $1005-1016$.

8. Kwon H-C, Oh SY, Lee S, Kim S-H, Kim H-J. Bevacizumab plus infusional 5-fluorouracil, leucovorin and irinotecan for advanced colorectal cancer that progressed after oxaliplatin and irinotecan chemotherapy: A pilot study. World J Gastroenterol. 2007;13(46): 6231-6235.

9. Soltau J, Drevs J. Mode of action and clinical impact of VEGF signaling inhibitors. Expert Rev Anticancer Ther. 2009;9(5):649-662.

10. Li MY, Lv YC, Tong LJ, et al. DW10075, a novel selective and smallmolecule inhibitor of VEGFR, exhibits antitumor activities both in vitro and in vivo. Acta Pharmacol Sin. 2016;37:398.

11. Zhu AX, Rosmorduc O, Evans TRJ, et al. SEARCH: A Phase III, Randomized, Double-Blind, Placebo-Controlled Trial of Sorafenib Plus Erlotinib in Patients With Advanced Hepatocellular Carcinoma. J Clin Oncol. 2015;33(6):559-566.

12. Michaelson MD, Oudard S, Ou Y-C, et al. Randomized, PlaceboControlled, Phase III Trial of Sunitinib Plus Prednisone Versus Prednisone Alone in Progressive, Metastatic, Castration-Resistant Prostate Cancer. J Clin Oncol. 2014;32(2):76-82.

13. Tian S, Quan H, Xie C, et al. YN968D1 is a novel and selective inhibitor of vascular endothelial growth factor receptor-2 tyrosine kinase with potent activity in vitro and in vivo. Cancer Sci. 2011;102(7):1374-1380.

14. Li J, Qin S, Xu J, et al. Randomized, Double-Blind, Placebo-Controlled Phase III Trial of Apatinib in Patients With Chemotherapy-Refractory Advanced or Metastatic Adenocarcinoma of the Stomach or Gastroesophageal Junction. J Clin Oncol. 2016;34(13):1448-1454.

15. Jin Z, Cheng X, Feng H, et al. Apatinib Inhibits Angiogenesis Via Suppressing Akt/GSK3ß/ANG Signaling Pathway in Anaplastic Thyroid Cancer. Cell Physiol Biochem. 2017;44(4):1471-1484.

16. Hu X, Cao J, Hu W, et al. Multicenter phase II study of apatinib in nontriple-negative metastatic breast cancer. BMC Cancer. 2014;14:820.

17. Langer CJ, Mok T, Postmus PE. Targeted agents in the third-/fourthline treatment of patients with advanced (stage III/IV) non-small cell lung cancer (NSCLC). Cancer Treat Rev. 2013;39(3):252-260.

18. Gou M, Si H, Zhang Y, et al. Efficacy and safety of apatinib in patients with previously treated metastatic colorectal cancer: a real-world retrospective study. Sci Rep. 2018;8(1):4602.

19. Prins MJ, Verhage RJ, ten Kate FJ, van Hillegersberg R. Cyclooxygenase isoenzyme-2 and vascular endothelial growth factor are associated with poor prognosis in esophageal adenocarcinoma. J Gastrointest Surg. 2012;16(5):956-966.

20. Fontana E, Sclafani F, Cunningham D. Anti-angiogenic therapies for advanced esophago-gastric cancer. Indian J Med Paediatr Oncol. 2014; 35(4):253-262.

21. Ferrara N. The Biology of Vascular Endothelial Growth Factor. In: Maragoudakis ME, editor. Molecular, Cellular, and Clinical Aspects of Angiogenesis. Boston, MA: Springer; 1996:73-83.

22. Ferrara N, Gerber H-P, LeCouter J. The biology of VEGF and its receptors. Nat Med. 2003;9:669. 
23. Bromann PA, Korkaya H, Courtneidge SA. The interplay between Src family kinases and receptor tyrosine kinases. Oncogene. 2004;23: 7957-7968.

24. Smith N, Baker D, James N, et al. Vascular endothelial growth factor receptors VEGFR-2 and VEGFR-3 are localized primarily to the vasculature in human primary solid cancers. Clin Cancer Res. 2010;16(14):3548-3561.

25. Yang L, Zhu H, Luo P, Chen S, Xu Y, Wang C. Apatinib mesylate tablet in the treatment of advanced malignant melanoma. Onco Targets Ther. 2018;11:5333-5338.

26. Yu WC, Zhang KZ, Chen SG, Liu WF. Efficacy and Safety of apatinib in patients with intermediate/advanced hepatocellular carcinoma: A prospective observation study. Medicine (Baltimore). 2018; 97(3):e9704.
27. Cheng H, Sun A, Guo Q, Zhang Y. Efficacy and safety of apatinib combined with chemotherapy for the treatment of advanced gastric cancer in the Chinese population: a systematic review and meta-analysis. Drug Des Devel Ther. 2018;12:2173-2183.

28. Li J, Wang L. Efficacy and safety of apatinib treatment for advanced esophageal squamous cell carcinoma. Onco Targets Therapy. 2017; 10:3965-3969.

29. Liu L, Wu N, Li J. Novel targeted agents for gastric cancer. J Hematol Oncol. 2012;5(1):31.

30. Fan M, Zhang J, Wang Z, et al. Phosphorylated VEGFR2 and hypertension: potential biomarkers to indicate VEGF-dependency of advanced breast cancer in anti-angiogenic therapy. Breast Cancer Res Treat. 2014; 143(1):141-151.

\section{Publish your work in this journal}

OncoTargets and Therapy is an international, peer-reviewed, open access journal focusing on the pathological basis of all cancers, potential targets for therapy and treatment protocols employed to improve the management of cancer patients. The journal also focuses on the impact of management programs and new therapeutic agents and protocols on

\section{Dovepress}

patient perspectives such as quality of life, adherence and satisfaction. The manuscript management system is completely online and includes a very quick and fair peer-review system, which is all easy to use. Visit http://www.dovepress.com/testimonials.php to read real quotes from published authors.

Submit your manuscript here: http://www.dovepress.com/oncotargets-and-therapy-journal 